Radiologe 2011 · 51:1013

DOI 10.1007/s00117-011-2260-5

Online publiziert: 27. November 2011

(c) Springer-Verlag 2011

\author{
M. Reiser ${ }^{1} \cdot$ C. Herold ${ }^{2}$ \\ ${ }^{1}$ Institut für Klinische Radiologie, Klinikum der \\ Ludwig-Maximilians-Universität, Campus Innenstadt, München \\ 2 Universitätsklinik für Radiodiagnostik, Medizinische Universität Wien
}

\title{
Europäisches Diplom für Radiologie
}

Im Beitrag von Dr. Éamann Breatnach auf Seite 1058 wird die Einführung des Europäischen Diploms für Radiologie dargestellt. Auf dem European Congress of Radiology (ECR) 2011 fand die erste Prüfung statt. Für die Radiologen im deutschsprachigen Raum, insbesondere die jungen Kollegen, stellt sich sicherlich die Frage, welche Ziele damit verfolgt werden und ob es für sie wichtig oder sogar notwendig ist, dieses Diplom zu erwerben.

Zunächst ist festzustellen, dass dieses Diplom in Deutschland keine rechtliche Konsequenz hat und die Anerkennungen des Facharztes durch die Landesärztekammern juristisch verbindlich sind.

In einigen anderen Fächern hat sich ein Europäisches Diplom in Ergänzung zum deutschen Facharzt etabliert. Zahlreiche junge Kollegen absolvieren die europäischen Prüfungen, oft im Zuge der Vorbereitungen für die deutsche Facharztprüfung. Auch diverse Subspezialitäten, wie die Interventionelle Radiologie (CIRSE), verleihen nach erfolgreicher Prüfung ein Europäisches Diplom.

Die bloße Tatsache, dass auch in anderen Fächern ein Europäisches Diplom eingeführt wurde, ist aber vermutlich keine ausreichende Motivation, sich dieser Mühe zu unterziehen. Ungeachtet der aktuellen Finanzkrise im Euroraum schreiten die Integration Europas und die Mobilität der Angehörigen zahlreicher Berufe fort. Weniger in Deutschland, dafür aber in anderen Ländern spielt die teleradiologische Befundung über die Grenzen der Nationalstaaten hinweg eine zunehmende Rolle. Gleichzeitig sind die Voraussetzungen, die erfüllt sein müssen, um als Facharzt zu praktizieren, sehr unterschiedlich. Das Europäische Diplom kann dazu beitra- gen, einen gleichmäßig hohen Standard der Qualifikation der Radiologen in Europa zu gewährleisten und damit die Sicherheit für unsere Patienten zu verbessern. Darüber hinaus kann die Tätigkeit im Ausland die Anerkennung einer Weiterbildung in Deutschland, Österreich oder in der Schweiz erleichtern. In verschiedenen europäischen Ländern, die keine formale Facharztprüfung eingeführt haben, wird schon jetzt überlegt, ob das Europäische Diplom als rechtlich verbindlich anerkannt werden kann.

Dr. Breatnach hat in seinem Beitrag die Entstehungsgeschichte, die Inhalte und die Art und Weise der Prüfung sowie die möglichen Zukunftsperspektiven für die Leser von Der Radiologe zusammengefasst. Wir möchten Ihnen die Lektüre dieses Artikels ans Herz legen und freuen uns sehr, wenn die deutschsprachigen Radiologen sich dieser Frage stellen und in einen breiten Diskurs eintreten.

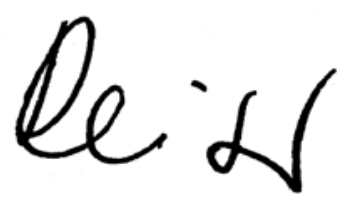

Prof. Dr. Maximilian Reiser

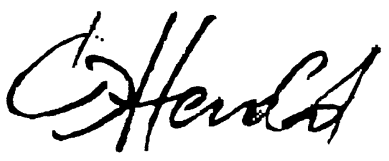

Prof. Dr. Christian Herold 